\title{
ESTRUCTURA DE IDIOMA EN QUE SE ESCRIBIERON LOS CÓDICES MAYAS
}

\author{
Ma. Cristina Álvarez
}

\section{Introducción}

Es importante para el estudio del descifre de la escritura maya tratar de determinar, sólo como punto de partida, la estructura del idioma empleado en la escritura glífica. El estudio estructural debe comprender los tres niveles lingüísticos fonológico, morfológico y sintático.

Todo idioma puede expresarse en forma oral y en forma escrita: en un momento dado, en cualquier fecha que se proponga, por la estructura del idioma deben corresponder la forma oral y la forma escrita.

Es necesario tener presente que el idioma empleado por los sacerdotes mayas, que se conservaba en forma escrita en los códices y se expresaba en forma oral, empleaba términos y simbolismos especiales; para poderlos entender, y sobre todo escribir, se necesitaba una preparación especializada que no tenía la gente del pueblo.

A esta "Forma" del lenguaje empleada por los sacerdotes se le llama "Código" para diferenciarla de la forma oral y la forma escrita que venimos utilizando en este estudio.

Este "Código" no debe haber cambiado mucho debido a la función socio-religiosa tan importante que tenía, ya que se empleaba para establecer comunicación con los dioses, donadores de beneficios o maleficios, y para las necesidades del pueblo en general.

El éxito o fracaso de esta comunicación dependía de la cuidadosa realización del ritual y de que las plegarias fueran dichas sin alterar el texto.

Estamos suponiendo que existía, y de hecho así sucedía, un "Código Popular", empleado para comunicarse los acontecimientos de la vida diaria, y un "Código Sacerdotal", empleado en el ritual; el sacerdote maya tenía el conocimiento de los dos Códigos. 
Por ejemplo el "Lenguaje de Zuyua" (Cap. IX de El Libro de Chilam Balam de Chumayel),* empleado por los sacerdotes mayas para hacer un examen a los aspirantes a batab (gobernadores) implica un conocimiento especializado no sólo del idioma sino también del ritual y la simbología empleada en él.

Ejemplos de los dos Códigos:

\section{a) Código Sacerdotal}

El halach uinic pide su comida a los aspirantes: talez kin mehene, ca a lathab tin plato. ti ch'icaan lanza, caanil cruz tan chumuc $u$ puczikal. ti ix culan yax balam yokol kin ukic u kikele.*

"Traedme el Sol, hijos míos, para ponerlo en mi plato. Hincada ha de tener la lanza de la alta cruz en el centro de su corazón a donde tiene asentado a yax bolon (sic), jaguar verde, bebiendo su sangre".

"Esto es lo que pide: el Sol es un gran huevo frito, y la lanza con la alta cruz hincada en su corazón a que se refiere es la bendición, y el jaguar verde sentado encima bebiendo sangre, es el chile verde cuando comienza a ponerse colorado".**

En resumen, lo que se pide es un huevo frito con salsa verde.

\section{b) Código Popular}

Cuando el sacerdote pide, en sus comidas habituales, un huevo frito con salsa, no lo pediría empleando el Código Sacerdotal (la forma anterior), sino empleando el Código popular y claramente pediría un tzah hee con u kaab ic (huevo frito con salsa de chile).

Los dos Códigos siguen las reglas estructurales del idioma y la misma semántica, la diferencia está en el simbolismo empleado en el Código Sacerdotal.

Todo idioma evoluciona en el transcurso del tiempo; pero el idioma empleado en el Código Sacerdotal tiende a hacerlo con más lentitud que el idioma empleado en el Código Popular.

De acuerdo con los estudios de Swadesh, ${ }^{*}$ todo idioma tiende a conservar un $86 \%$ de su léxico, más o menos, y a cambiar aproximadamente un $14 \%$, más o menos, en un periodo de mil años. En cambio los idiomas rituales, en los mismos mil sólo cambian $\pm 3 \%$.

* Roys, R. 1967.

** Barrera Vázquez, 1972. 
Por lo tanto, partiendo de un mismo Código, tuvieron evolución distinta el Código Sacerdotal y el Código Popular, de manera que, en un momento dado, el Código Sacerdotal resultó incomprensible para el pueblo en general.

La evolución del Código Sacerdotal terminó en el momento de la conquista del área maya realizada por los españoles; en cambio, la conquista propició una evolución rápida (relativamente) y particular de cada uno de los idiomas que forman la famiila maya.

De manera que aunque actualmente se encuentren grandes o pequeñas diferencias entre los idiomas de esta familia, el Código Sacerdotal quedó relativamente estático en el momento de la conquista.

El estudio se inició tomando como punto de partida los idiomas modernos de la familia maya, para comprender el funcionamiento de los procesos lingüísticos en los tres niveles: fonológico, morfológico y sintáctico de los idiomas de la familia maya, especialmente de los idiomas de la división Winik (Clasificación de Swadesh); pero la aplicación de los resultados debe hacerse cuidadosamente de acuerdo con los textos rituales.

Todo lo expuesto anteriormente los podemos representar gráficamente con dos líneas, una representa al Código Popular y la otra al Código Sacerdotal; el punto de partida de estas dos líneas indica que, posiblemente en una fecha muy lejana, los dos Códigos, el Sacerdotal y el Popular, eran el mismo. La fecha 500 d.C. que se emplea, es la fecha en la que Graham* considera la existencia de un sistema calendárico y una escritura glífica plenamente desarrolladas atribuíbles a los idiomas de las Tierras Bajas; fecha en que seguramente los dos Códigos (Popular y Sacerdotal) ya eran diferentes.

500 d.C.

1545

Código Sacerdotal

1975

Código Popular

\section{ESTUDIO}

El estudio lingüístico que puede ser de gran utilidad para el descifre de la escritura maya es aquel que en tiempo y espacio corresponda, más o menos, a la época y a la zona en la que se desarrolló la cultura del Periodo Clásico Maya; época del calendario y escritura glífica plenamente desarrolladas.

* Swadesh. Lengua y Cultura, 1960.

* Graham, 1971. 
Swadesh * clasifica a los idiomas de la familia maya en: la división Inik (huasteco y cotoque o chicomucelteco) la división Winik (todos los demás idiomas que forman esta familia maya); la investigación sólo se hizo de los idiomas que forman la división que ocupan el área maya, estudio que de ninguna manera nos lleva hasta el protoidioma.

Se encontró que entre los grupos de idiomas que forman la división Winik existen las suficientes diferencias que permiten subdividirlos en idiomas que ocupan la región de las Tierras Altas e idiomas que ocupan la región de las Tierras Bajas, del área maya. No es posible delimitar con una línea cortante los idiomas de estas dos regiones, ya que los procesos lingüísticos se manifiestan como "oleajes" de distintos alcances que parecen partir de los idiomas de las Tierras Altas hasta llegar a los idiomas de las Tierras Bajas.

Se quiere dejar bien aclarado que, cuando se habla de idiomas de las Tierras Altas e idiomas de las Tierras Bajas es sólo una forma de denominarlos con términos bien conocidos, aunque las regiones geográficas no correspondan exactamente a las zonas lingüísticas.

\section{Fonología}

El estudio fonológico de los idiomas de la división Winik se hizo tomando como base los fonemas ocluyentes. El Primer Cuadro corresponde a los idiomas que menor número de fonemas ocluyentes presenta y en el Cuadro 4 están los idiomas que tienen el mayor número de fonemas ocluyentes.

\section{Cuadro 1}

\section{FONEMAS BÁSICOS}

$\begin{array}{llllll}\text { simples: } & \mathrm{p} & \mathrm{t} & \mathrm{tz} & \mathrm{ch} & \mathrm{k} \\ \text { glotalizados: } & \mathrm{p} & \mathrm{t}^{\prime} & \mathrm{dz} & \mathrm{ch} & \mathrm{k}\end{array}$

Fonemas que se encuentran en el cuadro fonológico de los idiomas: yucateco, lacandón, mopán, itzá, chol, chontal, tzeltal, tzotzil, tojolabal, chuj, motocintleco, tuzanteco y uspanteco.

* Swadesh, 1966. 


\section{Guadro 2}

$\begin{array}{lllllll}\text { simples: } & \text { p } & \text { t } & \text { tz } & \text { ch } & \text { k } & \text { q } \\ \text { glotalizados: } & \text { p' } & t^{\prime} & \text { dz } & \text { ch' } & \text { k' } & \text { q' }\end{array}$

Fonemas que presentan en su cuadro fonológico los idiomas: huasteco, cotoque, achí, quiché, cakchiquel, tzutujil, kekchí y chortí.

\section{Guadro 3}

simples: $\quad \mathrm{p} \quad \mathrm{t} \quad \mathrm{tz} \quad \mathrm{ch} \quad \mathrm{k}$ ky $\mathrm{q}$

glotalizados: p' t' $d z \quad c h$ ' k' ky' q'

Fonemas que se encuentran en el cuadro fonol6gico de los idiomas: pocomán, pocomchí y kanjobal.

\section{Guadro 4}

$\begin{array}{lllllllll}\text { simples: } & \text { p } & \text { t } & \text { tz } & \text { ch } & \text { c } & \text { k } & \text { ky } & \text { q } \\ \text { glotalizados: } & \text { p' } & \text { t' } & \text { dz } & \text { ch' } & \text { c' } & \text { k' } & \text { ky' } & \text { q' }\end{array}$

Fonemas que se presentan en el cuadro fonológico de los idiomas ixil, jacalteco, aguacateco y mam.

Para representar gráficamente la distribución de los fonemas ocluyentes se elaboró un diagrama de Venn: el diagrama consta de varios círculos concéntricos en el que los idiomas que tienen el menor número de fonemas consonánticos ocluyentes ocupa el centro (número 1), y los idiomas que tienen el mayor número de fonemas consonánticos ocluyentes ocupa el círculo exterior (número 4). (Cf. el diagrama).

Los idiomas de las Tierras Bajas corresponden a los idiomas que presenta el cuadro fonológico $1 \mathrm{y}$ algunos presentan los fonemas del Cuadro 2; los idiomas de las Tierras Altas presentan los fonemas indicados en el Cuadro 4, y algunos idiomas tienen los fonemas del Cuadro 3.

Los idiomas cuyos fonemas se encuentran contenidos en los Cuadros 2 y 3 podemos considerarlos, en cierto modo, de transición; pero los idiomas del Cuadro 2 se relacionan más con los idiomas de las Tierras Bajas y los idiomas que tienen los fonemas del Cuadro 3 se relacionan más con los idiomas de las Tierras Altas.

Se presenta un mapa de distribución de los fonemas consonán- 
ticos ocluyentes de los idiomas de la división Winik de la familia maya que ocupa esta área. (Cf. mapa 1).

\section{Morfclogía}

A la manera como se estructuran los elementos de una palabra se le llama morfología; y es aquí en donde se encuentran diferencias tanto en la función, como en la posición y fonemas de los morfemas que estructuran una palabra.

Lo importante para nuestro trabajo es determinar en qué consisten las diferencias que separan a los grupos de idiomas de esta división y a cuáles grupos de idiomas unen esas mismas diferencias.

Los elementos constitutivos de la palabra son: raíz, afijos y clíticos. Para nuestros propósitos resultaron muy productivas las diferencias que presentaron los afijos, tanto en los fonemas que los estructuran, como la posición que ocupan en relación con la raíz o radical y la función que desempeñan.

Aquí sólo mencionaremos aquellos afijos que tienen las diferencias lo suficientemente claras para considerar seriamente la posibilidad de subdividir a los idiomas de la división Winik en idiomas de las Tierras Altas e idiomas de las Tierras Bajas.

\section{1) Pronombres}

Existen dos series básicas de pronombres empleados en todos los idiomas de la división Winik: Clase I y Clase II. Los pronombres de la Clase I funcionan como pronombres posesivos de un nombre, y sujeto con un verbo transitivo; los pronombres de la clase II funcionan como sujetos de verbos intransitivos o pronombres objeto con los verbos transitivos.

\section{a) Posición de los pronombres}

Los pronombres de la Clase II funcionan como pronombres objeto con una raíz verbal transitiva y como pronombres sujeto de una raíz verbal intransitiva; con una raíz nominal funcionan como estativos.

Estos pronombres de la clase II son prefijos (de segunda posición antes de la raíz) en los idiomas que ocupan la región de las Tierras Altas y son pronombres sufijos (última posición después de la raíz) en los idiomas que ocupan la región de las Tierras Bajas.

La diferencia la encontramos en la posición que ocupan los pronombres de la Clase II en relación con la raíz. (Cf. mapa 2). 
Los pronombres de la Clase I funcionan como pronombres posesivos con una raíz nominal y como pronombres sujeto con una raíz verbal transitiva. Estos pronombres son prefijos (de primera posiciión antes de una raíz) en todos los idiomas de la división Winik.

\section{b) Fonemas de los pronombres}

El contraste que puede establecerse con los pronombres de la Clase I, entre los idliomas de las Tierras Bajas y ls idiomas de las Tierras Altas, es por los fonemas que tienen los pronombres de las terceras personas: singular y plural.

Las Terceras personas gramaticales (singular y plural) son importante; se encontró que en los textos coloniales de El Libro de Chilam Balam de Chaumayel alcanzan un alto porcentaje (82\%) de frecuencia en relación con los pronombres de las demás personas gramaticales; por otra parte, existen textos del mismo Libro del Chilam Balam de Chumayel que están escritos empleando solamente las terceras personas gramaticales (él y ellos).

En el Quiché Clásico estudiado por Edmonson* se menciona que en estos textos se emplea la tercera persona gramatical.

Un proceso o cambio lingüístico alcanza a algunos idiomas, y no a todos, por lo que al presentar las terceras personas gramaticales del singular y las del plural se observará que los cambios lingüísticos no se encuentran en los mismos idiomas.

Los idiomas de las Tierras Bajas tienen para estas personas a ' $u$ "él" y $u-\ldots-o b$ (morfema discontinuo) "ellos". Los idiomas de las Tierras Altas tienen otros fonemas para estos pronombres.

\begin{tabular}{|c|c|c|c|}
\hline Singular: & él & Plural: & los \\
\hline Yucateco & $\mathrm{u}-$ & Yucateco & $\mathrm{u}-\ldots-\mathrm{ob}$ \\
\hline Lacandón & $\mathrm{u}-$ & Lacandón & $\mathrm{u}-\ldots-\mathrm{ob}$ \\
\hline Itzá & $\mathrm{u}-$ & Itzá & $\mathrm{u}-\ldots-\mathrm{ob}$ \\
\hline Mopán & $\mathrm{u}-$ & Mopán & $\mathrm{u}-\ldots-\mathrm{ob}$ \\
\hline Chortí & $\mathrm{u}-$ & Chortí & $\mathrm{u}-\ldots-\mathrm{ob}$ \\
\hline Chontal & $\mathrm{u}-$ & Chontal & $\mathrm{u}-\ldots-\mathrm{ob}$ \\
\hline & & Chol & $\mathrm{u}-\ldots-\mathrm{ob}$ \\
\hline & & Tzeltal & $\mathrm{u}-\ldots-\mathrm{ob}$ \\
\hline & & Tzoltzil & $\mathrm{u}-\ldots-\mathrm{ob}$ \\
\hline
\end{tabular}

Achí u-

* Edmonson, M. 1971. 
c) Proceso de sistematización de los pronombres

Entre los pronombres de la Clase I existe un proceso de sistematización; este proceso corresponde a la tendencia de reducir o normalizar los pronombres del plural con los del singular; por ejemplo, el pronombre que se presenta en la segunda persona singular (tú) se puede presentar en el plural más un sufijo pluralizador que marque la diferencia.

A los distintas formas de sistematización se les llamó "grados" con un número.

Los idiomas de las Tierras Altas tienen distintos grados de sistematización; en cambio en los idiomas de las Tierras Bajas el proceso de sistematización de los pronombres de la Clase I corresponde al grado 3 ; en el grado 3 se encuentran los siguientes pronombres:

$\begin{array}{llll}\text { in- } & \text { yo } & & \\ a- & \text { tu } & a-\ldots-e x & \text { ustedes } \\ u- & \text { él } & u-\ldots-o b & \text { ellos } \\ k- & \text { nosotros } & & \end{array}$

Cuatro pronombres con un solo morfema y dos pronombres del plural con los mismos fonemas que los pronombres del singular, más un sufijo pluralizador.

Todas estas diferencias y semejanzas se concentraron en el Cuadro 5 , de manera que el lector pueda apreciar claramente la posición de los pronombres de la Clase II; los diferentes fonemas que tienen los pronombres de las terceras personas gramaticales de la Clase I y los distintos grados del proceso de sistematización.

\section{2) sufijos verbales}

Los sufijos $-V h /-V$ y los sufijos $-V k /-V q$ ( $V$ corresponde a cualquier vocal que se presente en estos sufijos) que se encuentran en las formas verbales, por la función que desempeñan, permiten establecer un contraste entre los idiomas de las Tierras Altas y los idiomas de las Tierras Bajas.

El sufijo $-V h$ es marcador de verbos en activo de los verbos transitivos en los idiomas de las Tierras Altas y es marcador de pasado en los idiomas de las Tierras Bajas. 
ESTRUGTURA DE IDIOMA EN QUE SE ESCRIBIERON LOS...

Tierras Altas:

Contrastes
Traducción

al Español
Semejanzas

_ $V h$ marcador de activo verbos transitivos tiempo pasado

Tierras Bajas:

-ah marcador de pasado verbos transitivos tiempo pasado

El sufijo $-V$ es marcador de activo de los verbos intransitivos en los idiomas de las Tierras Altas; en los idiomas de las Tierras Bajas es difícil determinar la función de este sufijo; en algunos idiomas de las Tierras Bajas no aparece este sufijo con esta función de activo como en el yucateco, lacandón, etc.; en algunos idiomas como en el chol de Tila, el sufijo $-V$ marca pasado o acción completa de los verbos transitivos. En el Chontal y el chortí el sufijo $-V$ es marcador de activo de verbos transitivos.

Sufijos $-V q /-V k$. En los idiomas de las Tierras Altas estos sufijos verbales funcionan como marcadores de aspecto definido $(-V q)$ y de aspecto indefinido de los verbos transitivos ( $-V k)$.

En los idiomas de las Tierras Bajas no existe el fonema $q$ y su correspondiente glotalizador $q^{\prime}$; que es sustituido por el fonema $k$ y su correspondiente glotalizador $k^{\prime}$ por lo que los sufijos $-V q$ y $-V k$ que se presentan en las formas verbales de los idiomas de las Tierras Altas se reducen a una sola forma $\_V k$ en los idiomas de las Tierras Bajas. El morfema _ $V k$ funciona como marcador de tiempo presente y futuro de los verbos transitivos o intransitivos.

Parece notarse cierta relación entre tiempo presente con el aspecto definido y tiempo futuro con el aspecto indefinido.

Tierras Bajas:

\begin{tabular}{|c|c|c|}
\hline Yucateco & $\begin{array}{l}-\mathrm{ik} \\
-\mathrm{Vk}\end{array}$ & $\begin{array}{l}\text { marcador de tiempo presente. } \\
\text { marcador de tiempo futuro. }\end{array}$ \\
\hline Lacandón & $\begin{array}{l}\text {-ik } \\
-\mathrm{Vk}\end{array}$ & $\begin{array}{l}\text { marcador de tiempo presente } \\
\text { marcador de tiempo futuro. }\end{array}$ \\
\hline Itzá & $\begin{array}{l}-\mathrm{ik} \\
-\mathrm{Vk}\end{array}$ & $\begin{array}{l}\text { marcador de tiempo presente. } \\
\text { marcador de tiempo futuro. }\end{array}$ \\
\hline Мо & $-\mathrm{ik}$ & marcador de continuativo. \\
\hline
\end{tabular}

Tierras Altas:

Aguacateca $-\mathrm{q} /-\mathrm{Vq}$ marcador de intransitivo

Achí $\quad-\mathrm{Vk}$ marcador de intransitivo. Aspecto indefinido. -Vq marcador de intransitivo. Aspecto definido. 
Quiché - Vk marcador de intransitivo. Aspecto indefinido.

$\begin{array}{lll} & -\mathrm{Vq} & \text { marcador de intransitivo. Aspecto definido. } \\ \text { Kekchí } & -\mathrm{ik} & \text { marcador de intransitivo. Aspecto indefinido. }\end{array}$

-ik marcador de intransitivo. Indefinido.

-ok marcador de intransitivo. Definido.

\section{Sintaxis}

Por sintaxis debe entenderse la relación que guardan entre sí las palabras al formar construcciones mayores (frase y oración). La palabra es la unidad mínima de la construcción y la oración es la construcción máxima; podemos considerar a la frase como una construcción intermedia entre la palabra y la oración. Por lo tanto la oración sencilla puede definirse como "una serie potencial de clases de frases (una o más) arregladas de acuerdo con un patrón característico. La clase de frase obligatoria es un predicado que puede estar formado por una frase verbal o por una frase sustantiva. La presencia o ausencia de ciertas clases de frases dependientes distinguen a los varios tipos de oraciones".*

Las fórmulas que se emplean en este estudio son variantes de las empleadas por Shaw y Neuenswander en su estudio del Achí.

Oraciones transitivas:

$$
\pm \mathrm{I} / \mathrm{T} \pm \mathrm{S}+\mathrm{Pt} \pm \mathrm{S} \pm \mathrm{O} \pm \mathrm{IO} \pm \mathrm{M} \pm \mathrm{T} / \mathrm{G} * *
$$

Oraciones intransitivas:

$$
\pm \mathrm{I} / \mathrm{T} \pm \mathrm{S}+\mathrm{Pi} \pm \mathrm{S} \pm \mathrm{L} \pm \mathrm{Ac} / \mathrm{C}
$$

Oración nominal:

$$
\text { Comentario + Tópico }
$$

* Shaw, Mary y Neuenswander, Helen, 1966.

* Notas de las abreviaturas.

$\mathrm{I}=$ Introductorio

$\mathrm{T}=$ Tiempo

$\mathrm{S}=$ Sujeto

$\mathrm{Pt}=$ Predicado transitivo

$\mathrm{O}=$ Objeto

$\mathrm{IO}=$ Objeto Indirecto

$\mathbf{M}=$ Modificador

$\mathbf{C}=$ Complemento

$\mathrm{Pi}=$ Predicado intransitivo

$\mathbf{L}=$ Locativo

Ac $=$ Acompañamiento 
ESTRUCTURA DE IDIOMA EN QUE SE ESCRIBIERON LOS...

El elemento que nunca puede faltar en la oración verbal es el predicado; los demás elementos son optativos como se indica en las fórmulas $( \pm)$.

Cada elemento o parte de la oración puede ser llenado con una palabra o con una frase.

A continuación se presenta la estructura de una frase:

Frase nominal:

$$
\pm \text { Partícula } \pm \text { Modificador }+ \text { nombre. }
$$

Frase predicante:

$$
\pm \text { Partícula } \pm \text { Modificador + Forma Verbal. }
$$

\section{Conclusiones}

Es frecuente encontrar en los textos con temas rituales oraciones construidas con predicados estructurados con un pronombre de la Clase I y una raíz sin sufijos, en la que es difícil determinar si se trata de un nombre poseído o de una forma verbal amplia o generalizada. De acuerdo con la anterior construcción podríamos decir que los Códices Mayas podían escribirse y leerse en cualquier idioma de la familia maya.

Los afijos, sobre todo las dos series de pronombres, por la posición que ocupan en relación con la raíz (prefijo o sufijo), por los fonemas que estructuran a los pronombres de la tercera persona gramatical (singular y plural) y el grado de sistematización de los pronombres, nos permiten subdividir a los idiomas de la división Winik en idiomas que ocupan la región de las Tierras Altas e idiomas que ocupan la región de las Tierras Bajas.

Los sufijos -Vh/-V y los sufijos -Vq/-Vk que presentan las formas verbales, también permiten establecer el contraste entre los idiomas de las Tierras Altas y los idiomas de las Tierras Bajas.

Los fonemas que presentan en su cuadro fonológico los idiomas de las Tierras Bajas son los contenidos en los Cuadros 1 y 2.

Por lo tanto, se propone que la estructura del idioma en que fueron escritos los Códices Mayas posiblemente tenga las siguientes características:

1) Las oraciones siguen un patrón general para todos los idiomas de la división Winik de la familia maya.

2) Las diferencias las encontramos en la frase predicante que bien puede ser: 
a) Una forma amplia o generalizada (la raíz sin los sufijos derivacionales).

b) Presentar los sufijos - ah para marcar la forma verbal en activo transitivo; o el sufijo $-V$ para los verbos en activo intransitivo.

c) Que las formas intransitivas tengan los sufijos $-V k /-V q$ para marcar los aspectos indefinido y definido.

3) Que los pronombres de tercera persona singular tengan la forma $u$ - "él" (incluyéndose los idiomas Achí y Quiché); y para el plural $u-\ldots-o b$ "ellos" (no se incluye al Achí y Quiché).

4) Que la fonología puede ser la que se presenta en los Cuadros 1 y 2 de los fonemos vocálicos esté la sexta vocal $(t)$.

Todo esto podrá ratificarse o rectificarse con estudios detallados de textos rituales de los idiomas de las Tierras Bajas, y deben incluirse los idiomas Achí y Quiché.

Falta por investigarse la alta sintaxis, semántica, conceptos y símbolo empleados en la escritura de los Códigos Mayas.

Por lo tanto, podemos concluir que la estructura del idioma empleado en los códigos mayas sigue las reglas de los idiomas de las Tierras Bajas; pero que en cuanto a semántica, conceptos y símbolos debe ser tratado como un idioma especial de las Tierras Bajas.

\section{OBRAS CONSULTADAS}

Álvarez, Ma. Cristina

1969 Descripción Estructural del Maya del Chilam Balam de Chumayel. Seminario de Estudios de la Escritura Maya. Coordinación de Humanidades. UNAM. Cuaderno 1. pp. 1-87. México.

1974 Textos Coloniales del Libro de Chilam Balam de Chumayel y Textos Glíficos del Códice de Dresde. Centro de Estudios Mayas. Coordinación de Humanidades. UNAM. México. Cuaderno 10. pp. 1-108.

Barthel, Thomas S.

1973 "Los Jeroglíficos Mayas, una Escritura de la Antigua América". En Guatemala Indígena. Instituto Nacional Indigenista. Editorial "Galindo" Guatemala, C.A. Volumen VIII, No. 1, pp. 69-80

Brasseur de Bourbourg, Charles B.

1961 Gramática de la Lengua Quiché. Ministerio de Educación Pública, Editorial "José de Pineda Ibarra". Guatemala, C.A. Publicaciones especiales del Instituto Indigenista Nacional. 
Bruce S., Roberto D.

1968 Gramática del Lacandón. Departamento de Investigaciones Antropológicas. Instituto Nacional de Ontropología e Historia. México. Publicación 21. pp. 1-151.

Cazes, Daniel

1968 "Frecuencia de algunos elementos lingüísticos en el Chilam Balam de Chumayel". En Estudios de Cultura Maya. Centro de Estudios Mayas. Coordinación de Humanidades. UNAM. México. pp. 255-288.

Church, Clarence y Kay

1966 "Gramática Jacalteca". En Lenguas de Guatemala. Seminario de Integración Social Guatemalteca. Ministerio de Educación. Guatemala, C.A. Vol. 20, pp. 293-310.

DAKIN, K.

1974 Kanjobal. Dialectos de Santa Eulalia y Saloma. Apuntes Preliminares. Proyecto Lingüístico Francisco Marroquín. Guaternala, C.A.

Eachus, Francís y Carlson, Ruth

1966 "Bosquejo Gramatical Kekchí". En Lenguas de Guatemala. Seminario de Integración Social Guatemalteca. Ministerio de Educación. Guatemala, C.A. Vol. 20, pp. 163-177.

Edmonson, Munro S.

1964 "Historia de las Tierras Altas Mayas seg\$n documentos Indígenas". En Desarrollo Cultural de los Mayas. Seminario de Cultura Maya. Facultad de Filosofía y Letras. UNAM. M!xico, pp. 255-278.

1967 "Classical Quiché". En Handbook of Middle American Indians. Linguistics. University of Texas Press Austin. Vol. 5, pp. 249-268.

Elliot, Raymond y Helen

1966 "Gramática Mam". En Lenguas de Guatemala. Seminario de Integración Social Guatemalteca. Ministerio de Educación. Guatemala, C.A. Vol. 20, pp. 191-199.

Elliot, Raymond L.

1973 "Notas sobre algunas características lingüísticas de idiomas indígenas". En Guatemala Indigena. Insituto Nacional Indigenista. Editorial "Galindo" Guatemala, C.A. Volumen VIII, No. 1 , pp. $69-80$.

Escalante, Roberto

1971 Análisis de Estructuras en el Códice de Dresde. Centro de Estudios Mayas. Coordinación de Humanidades. UNAM. México. Cuaderno 4, pp. 1-90. 
Fought, John G.

1972 Chortí, (mayan) Texts. Edited by Sarah S. Fought. University of Pennsylvania Press. Philadelphia. U.S.A., pp. 1-565.

Fox, David

1966 "Quiché" "Gramática". En Lenguas de Guatemala. Seminario de Integración Social Guatemalteca. Ministerio de Educación. Guatemala, C.A. Vol. 20, pp. 87-125.

Garicía de León, Antonio

1971 Los Elementos del Tzotzil Colonial y Moderno. Centro de Estudios Mayas. Coordinación de Humanidades. UNAM. México, pp. 1-107.

Graham, John

1971 "Sobre la Escritura Maya". En Desarrollo Cultural de los Mayas. Editado por Evon Z. Vogt y Alberto Ruz. Centro de Estudios Mayas. Coordinación de Humanidades. UNAM. México, pp. 257-272.

Guerrero, Javier

1971 Las Funciones de la Religi $n$ y la Magia en la Organización Social de los Antiguos Mayas. Tesis Profesional. Escuela Nacional de Antropología e Historia. México, pp. I-IV, 1-332.

Kaufman, Terrence

1967 Preliminary Mochó Vocabulary. Working Paper. Number 5. Laboratory for Language-Dehavior Research. University of California, Berkeley. U.S.A.

1971 "Materiales Lingüísticos para el Estudio de las Relaciones internas y externas de la Familia de Idiomas Mayanos". Editado en Desarrollo Cultural de los Mayas. Editado por Evon Z. Vogt y Alberto Ruz. Centro de Estudios Mayas. Coordinación de Humanidades. UNAM. México, pp. 81-136.

1972 El Proto-Tzeltal-Tzotzil. Fonología comparada y diccionario reconstruido. Centro de Estudios Mayas. Coordinación de Humanidades. UNAM. México. Cuaderno 5, pp. 1-161.

La Farge II, Olíver y Byers, Douglas

1931 "The Jacalteca Language". The Year Rearer's People. The Departament of Middle American Research. The Tulane University of Lousiana, New Orleans. U.S.A., pp. 247-294.

Marquina, Ignacio

1964 Arquitectura Prehispánica. Instituto Nacional de Antropología e Historia. Secretaría de Educación Pública. México, pp. I-XI, 1-1055.

Marvin, K. y Mayers, Marilyn A.

1966 "Sumario Gramatical Pocomchi". En Lenguas de Guatemala. Seminario de Integración Social Guatemalteca. Ministerio de Educación. Guatemala, C.A. Vol. 20, pp. 146-156. 
McArthur, Harry y Lucille

1966 "Gramática Aguacateca". En Lenguas de Guatemala. Seminario de Integración Social Guatemalteca. Ministerio de Educación. Guatemala, C.A. Vol. 20, pp. 220-235.

MaQuown, Norman

1967 "Classical Yucatec (Maya)". En Handbook of Middle American Indians. Linguistics. University of Texas Press Austin Vol. 5, pp. 201-248.

1971 "Los orígenes y la diferenciación de los Mayas según se infiere del estudio comparativo de las lenguas Mayanas". En Desarrollo Cultural de los Mayas. Editado por Evon Z. Vogt y Alberto Ruz. Centro de Estudios Mayas. Coordinación de Humanidades. UNAM. México, pp. 49-80.

OAKLey, Helen

1966 "Gramática Chortí. En Lenguas de Guatemala. Seminario de Integración Social Guatemalteca. Ministerio de Educación. Guatemala, C.A. Vol. 20, pp. 342-353.

Pérez, Benjamín

1968 "Fonología Preliminar del Chontal de Tabasco". En Boletín Informativo. Seminario de Estudios de la Escritura Maya. Coordinación de Humanidades. UNAM. México. Año 2, No. 2, Mayo, pp. 25-36.

Robles Uribe, Carlos

1962 Manual del Tzeltal (Gramática Tzeltal de Bachajon). Publicaciones de Antropoloogía. Lingüística. Universidad Iberoamericana. México, pp. 1-115.

ROWERo, MoIsÉS

1960 "Algunas observaciones sobre la dialectología Tzeltal". En Anales. Instituto Nacional de Antropología e Historia. Secretaría de Educación Pública. México. Tomo XIII. No. 42 de la Colección, pp. 207-216.

Roys, RALPH L.

1967 The Book of Chilam Balam de Chumayel. University of Oklahoma Press. Norman、 United States of America.

Sahumann G., Отто

1968 "Sobre el fonetismo vocálico en Pocomán". En Escritura Maya. Boletín Informativo. Seminario para el Estudio de la Escritura Maya. Coordinación de Humanidades. UNAM. México. Año 2, No. 2, p. 42.

1970 "La Posición del Tojolabal en la Familia Maya". En Escricritura Maya. Boletín Informativo. Centro de Estudios Mayas. Coordinación de Humanidades. UNAM. México. Año 4. No. 2. Octubre, pp. 4-9.

1971 Descripción Estructural del Maya Itzá del Petén, Guatemala, 
C. A. Centro de Estudios Mayas. Coordinación de Humanidades. UNAM. México. Cuaderno 6, pp. 1-134.

1973 La Lengua Chol de Tila (Chiapas). Centro de Estudios Mayas. Coordinación de Humanidades. UNAM. México. Cuaderno 8, pp. 7-113.

En prensa La Lengua Chortí. Centro de Estudios Mayas. Instituto de Filología. UNAM. México.

Shaw, Mary y Neuenswander, Helen

1966 "Achí. Gramática". En Lenguas de Guatemala. Seminario de Integración Social Guatemalteca. Ministerio de Educación. Guatemala, C.A. Vol. 20, pp. 87-124.

Smailus, Otwin

1969 "The Maya-Chontal Language of Acalan". En Escritura Maya. Boletín Informativo. Centro de Estudios Mayas. Coordinación de Humanidades. UNAM. México. Año 3. No. 3, pp. 1-24.

Swadesh, Mauricio

1966 "Interrelaciones de las Lenguas Mayanses". En Anales. Instituto Nacional de Antropología e Historia. Secretaría de Educación Pública. México. Tomo XIII. No. 42. de la Colección, pp. 231-268.

SYWulka, EDWard

1966 "Gramática Mam". En Lenguas de Guatemala. Seminario de Integración Social Guatemala. Ministerio de Educación. Guatemala, C.A. Vol. 20, pp. 255-277.

Thompson, J. Eríc

1972 Commentary on the Dresden Codex. American Philosophical Society. Independence Square. Philadelphia. U.S.A., pp. 1-156.

Ulrich, Matthew y Rosemary

1966 "Sumario Gramatical Mopán-Maya". En Lenguas de Guatemala. Seminario de Integración Social Guatemalteca. Ministerio de Educación. Guatemala, C.A. Vol. 20, pp. 370-382.

VÁzQuez, Esther

1968 "Fonolgía Preliminar del Pocomán Central". En Escritura Maya. Boletín Informativo. Seminario para el Estudio de la Escritura Maya. Año 2. No. 2, pp. 37-42.

Warkentin, Vrola y Whittaker, Arabelle

1970 "Tumbalá Chol Clause Structure". Linguistics. An International Review. Mounton. No. 60, pp. 74-110.

WILley, Gordon R.

1964 "An Archaeological frame of reference for Maya Culture History". En Desarrollo Cultural de los Mayas. Editado por Evon Z. Vogt y Alberto Ruz. Seminario de Cultura Maya. Facultad de Filosofía y Letras. UNAM. México, pp. 137-178. 
ESTRUCTURA DE IDIOMA EN QUE SE ESCRIBIERON LOS...

Williams, Kenneth y Barbara

1966 "Gram"tica Chuj". En Lenguas de Guatemala. Seminario de Integración Social Guatemalteca. Guatemala, C.A. Vol. 20, pp. $370-382$.

Barrera Vázquez, Alfredo y Rendón, Silva

1972 México. El Libro de los Libros de Chilam Balam. Fondo de Cultura Económica. Colección Popular. No. 42. México.

Swadesh, Mauricio

1960 Estudios sobre Lengua y Cultura. Acta Antropológica. 2a. época II-2. Escuela Nacional de Antropología e Historia. Sociedad de Alumnos. México.

\section{PERSONAS CONSULTADAS}

\section{Schumann Otto.}

Investigador del Centro de Estudios Mayas. Instituto de Investigaciones Filológicas. UNAM. México.

MAPA

El mapa que se utiliza en este estudio fue proporcionado por el Departamento de Lingüística del Insituto Nacional de Antropología e Historia. 
MAPA 1

DISTRIBUCION DE LOS FONEMAS CONSONANTICOS .

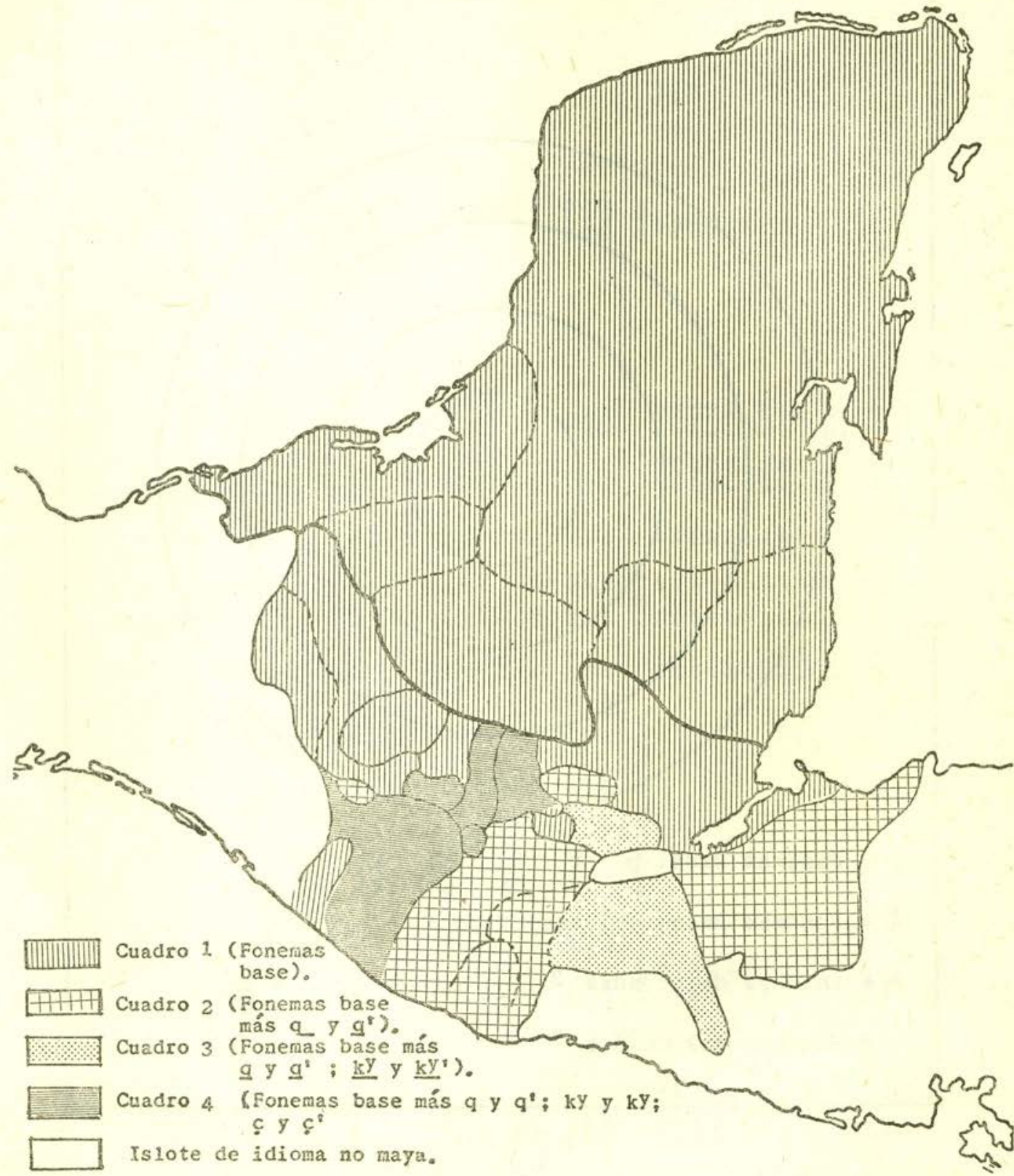

Estudios de Cultura Maya. Vol. X, 1976/7

Instituto de Investigaciones Filológicas/ Facultad de Filosofía y Letras

Centro de Estudios Mayas, UNAM

http://www.iifilologicas.unam.mx/estculmaya/ 


\section{MAPA 2.}

PRONOMBRES CLASE II.

Posición de los pronombres.

(en relación con la raíz).

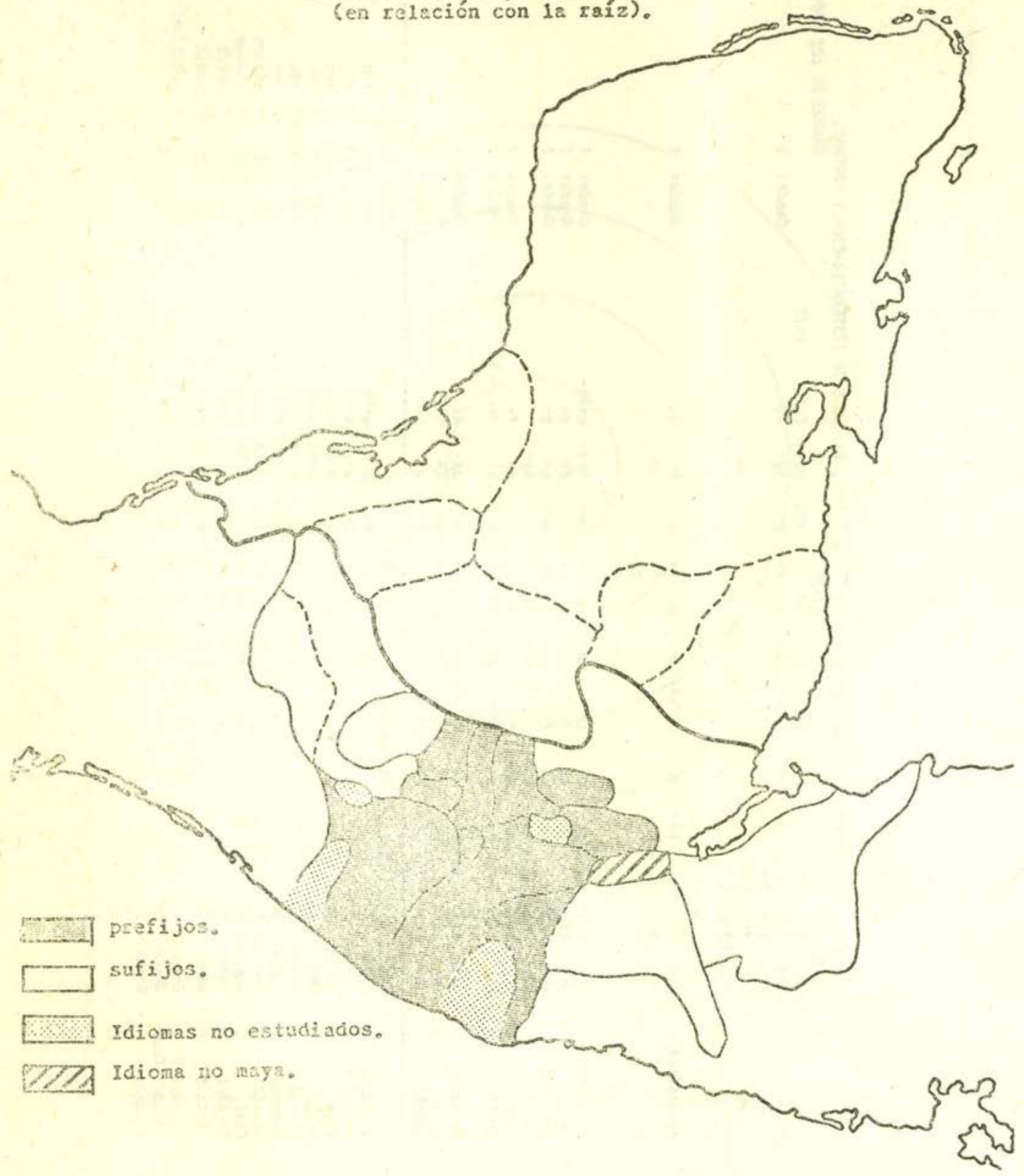

Estudios de Cultura Maya. Vol. X, 1976/7

Instituto de Investigaciones Filológicas/ Facultad de Filosofía y Letras Centro de Estudios Mayas, UNAM

http://www.iifilologicas.unam.mx/estculmaya/ 


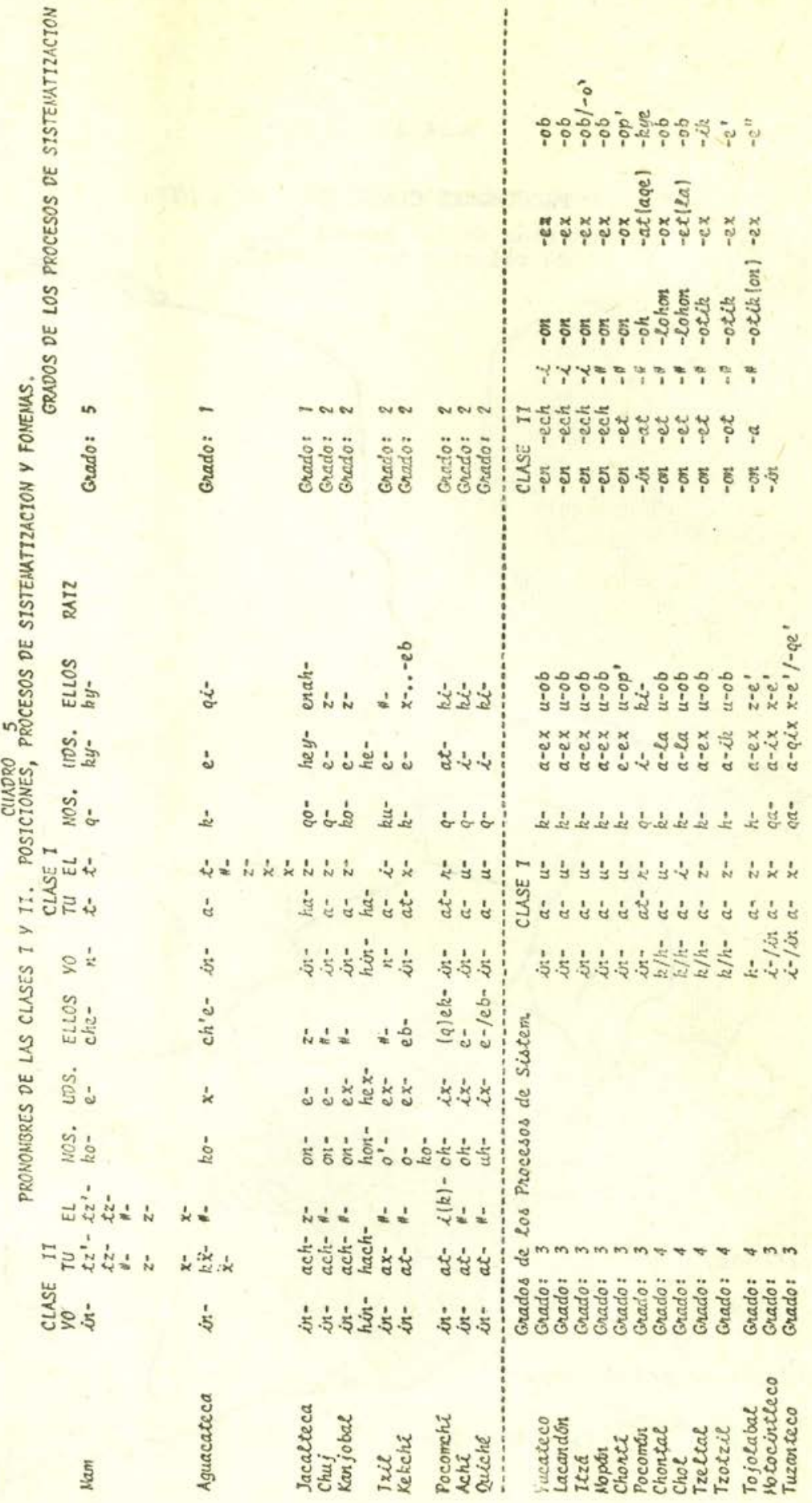




\section{DIAGRAMA DE VBNN.}

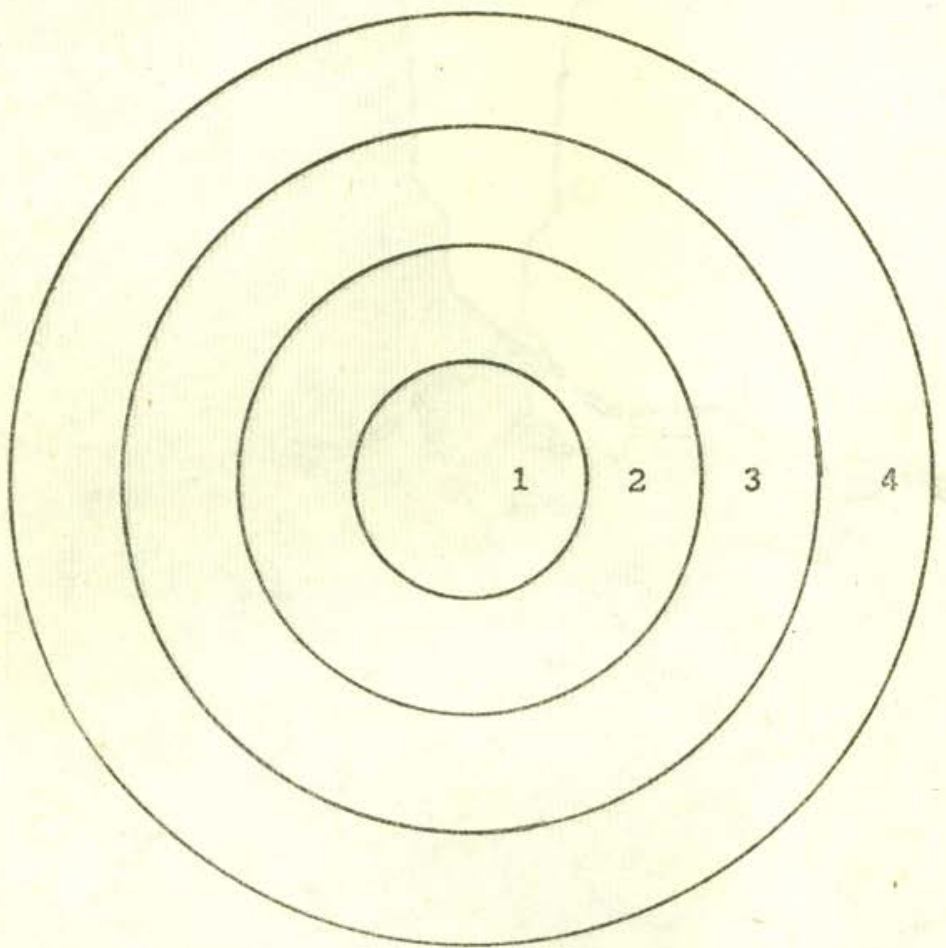

$1=$ Fonemas Base: $\begin{array}{lllll}p & t & t z & \mathrm{ch} & \mathrm{k} \\ \mathrm{p}^{*} & \hat{t}^{*} & \mathrm{dz} & \mathrm{ch} & \mathrm{k}^{*}\end{array}$

2 = Fonemas Base: del cuadro 1

3 = Fonemas del cuadro 2

$+k_{k y} y$

4 * Fonemas del cuadro 3 